Disponível em

http://www.anpad.org.br/rac

RAC, Rio de Janeiro, v. 20, n. 2, art. 5, pp. 216-237, Mar./Abr. 2016

http://dx.doi.org/10.1590/1982-7849rac2016140083

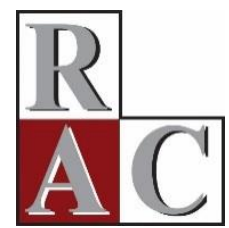

\title{
Percepção de Justiça nos Sistemas de Controle Gerencial Aumenta Comprometimento e Confiança dos Gestores?
}

Does the Perception of Fairness in Management Control Systems Increase Manager Commitment and Confidence?

Ilse Maria Beuren ${ }^{1}$

Luciana Klein ${ }^{2}$

Flávio Luiz Lara ${ }^{2}$

Lauro Brito de Almeida ${ }^{2}$

Universidade Federal de Santa Catarina ${ }^{1}$

Universidade Federal do Paraná ${ }^{2}$

Artigo recebido em 31.10.2014. Última versão recebida em 17.04.2015. Aprovado em 19.04.2015. Publicado online em 01.12.2015. 


\title{
Resumo
}

Este estudo objetiva verificar se a percepção de justiça em relação às dimensões dos Sistemas de Controle Gerencial aumenta o comprometimento e a confiança dos gestores. Para tal, foi realizada pesquisa de natureza descritiva por meio de levantamento, tendo como população as 500 empresas listadas entre as Melhores e Maiores da Revista Exame, das quais 180 aceitaram participar do estudo, e a amostra constituiu-se dos 67 respondentes. O instrumento de pesquisa utilizado compõe-se de 26 assertivas, com escalas de medidas intervalares Likert de sete pontos. As hipóteses enunciadas na pesquisa foram testadas com aplicação da técnica estatística de análise multivariada denominada Modelagem de Equações Estruturais. Os resultados da pesquisa demonstram que o princípio da controlabilidade e a utilização de múltiplos indicadores de desempenho aumentam a percepção de justiça distributiva e processual; a percepção de justiça das dimensões interacional e processual aumenta o comprometimento dos gestores; e a confiança dos gestores é influenciada pela percepção de justiça distributiva e interacional. Com base nesses resultados, conclui-se que o comprometimento e a confiança dos gestores podem ser fatores atitudinais e comportamentais preponderantes na diminuição de ações retaliatórias causadas pelo sentimento de injustiça organizacional.

Palavras-chave: justiça organizacional; sistemas de controle gerencial; comprometimento dos gestores; confiança dos gestores.

\begin{abstract}
The goal of this is study is to determine whether the perception of fairness regarding the dimensions of the Management Control Systems increases managers' commitment and confidence. Thus, a descriptive research was carried out through a survey of 500 companies listed among the Best and Biggest by Exame Magazine, from which 180 agreed to participate in the study. The final sample consisted of 67 respondents. The instrument used consists of 26 statements, with seven-point Likert interval scales of measurement. The assumptions made in the study were tested with the application of a statistical multivariate analysis technique called Structural Equation Modeling. The survey results show that the controllability principle and the use of multiple performance indicators increase the perception of distributive and procedural fairness; the perception of interactional and procedural fairness dimensions increase managers' commitment; and managers' confidence is influenced by perceptions of distributive and interactional fairness. Based on these results we conclude that managers' commitment and confidence can be prominent attitudinal and behavioral factors in reducing retaliatory actions caused by a sense of organizational unfairness.
\end{abstract}

Key words: organizational fairness; management control systems; managers' commitment; managers' confidence. 


\section{Introdução}

A justiça organizacional é construída socialmente e tem influência determinante nas atitudes e comportamentos emitidos no ambiente de trabalho (Cropanzano \& Folger, 1989; Skarlicki, Folger, \& Tesluk, 1999). Constitui-se, portanto, em um fenômeno humano complexo e multifacetado (Leventhal, Karuza, \& Fry, 1980) que abrange diversos domínios da vida e influencia ações individuais e coletivas. Dessa forma, a justiça organizacional deve ser entendida como a percepção de justiça pelos funcionários no que tange às relações de trabalho na organização (Parker \& Kohlmeyer, 2005).

De acordo com Folger e Cropanzano (1998), justiça organizacional se refere à percepção dos empregados sobre o que é justo ou injusto dentro das organizações em que trabalham. Omar (2006) adverte que, nos casos em que os empregados percebem como justo o tratamento da empresa, esta percepção contribuirá para terem atitudes positivas com o trabalho, os chefes e a organização como um todo, enquanto que, para os que consideram que estão sendo tratados injustamente, tal cognição gerará tensões, sentimentos de insatisfação e de desmotivação, absenteísmo, redução da qualidade do trabalho e perda de produtividade.

Pesquisas relativas à justiça organizacional têm ampliado o conhecimento sobre as diferentes formas de reações às injustiças percebidas e sobre os processos que deflagram comportamentos decorrentes. O estudo de Langevin e Mendoza (2013) aponta que os Sistemas de Controle Gerencial (SCG), quando considerados injustos, podem levar os gestores a terem comportamentos prejudiciais à empresa. Os autores advertem que ao invés de motivar, em algumas situações os SCG podem não contribuir à consecução dos objetivos da empresa e, dessa forma, diminuir o desempenho organizacional e a remuneração variável.

O princípio da controlabilidade propõe considerar na avaliação de desempenho dos gestores somente os elementos que eles conseguem controlar e o uso de múltiplos indicadores, financeiros e nãofinanceiros, alinhados com as atribuições dos gestores (Langevin \& Mendoza, 2013). Segundo os autores, essas dimensões possibilitam diminuir o sentimento de injustiça percebida pelos gestores, além de estarem diretamente ligadas ao aumento do nível de comprometimento organizacional e da confiança percebida.

De acordo com Mowday, Steers e Porter (1979), quando os gestores estão comprometidos com a organização, eles tendem a se identificar com ela e com os objetivos organizacionais e, por conseguinte, desejam manter sua vinculação profissional com a empresa. Portanto, quando os gestores possuem um forte comprometimento organizacional, serão menos inclinados a tomar decisões que possam ser prejudiciais à empresa.

A confiança nos incentivos transmite maior significado na percepção de controle em relação aos resultados. As percepções de controle são um componente essencial da justiça organizacional (Folger \& Greenberg, 1985). Alguns estudos demonstraram que, quando os gestores são recompensados pelo seu desempenho, é mais provável a percepção da equidade ou justiça (Greenberg, 1986, 1990). Outras pesquisas mostraram que todas as dimensões de justiça (distributiva, processual e interacional) estão correlacionadas com confiança (Ambrose \& Arnaud, 2005; Cropanzano, Bowen, \& Gilliland, 2007).

Whitener, Brodt, Korsgaard e Werner (1998) destacam que, a partir do momento em que os gestores confiam em seus superiores, estarão menos propensos a desenvolver comportamentos antiéticos em suas funções, uma vez que a confiança reflete uma expectativa ou crença de que a outra parte vai agir com benevolência. Todavia, os SCG podem induzir os gestores a comportamentos antiéticos (Langevin \& Mendoza, 2013), como a criação de folga orçamentária (Dunk, 1993) e a manipulação de dados (Defond \& Park, 1997; Merchant \& Rockness, 1994).

O pressuposto é que a justiça percebida nas dimensões (controlabilidade e múltiplos indicadores de desempenho) dos SCG (Langevin \& Mendoza, 2013) atua em dois níveis: (a) afeta a relação entre o empregado e a organização como um todo (comprometimento organizacional) (Mowday, Steers, \& 
Porter, 1979); e (b) influencia suas relações com seus superiores (confiança no supervisor) (Whitener, Brodt, Korsgaard, \& Werner, 1998). Ambos levam à maior congruência entre os objetivos organizacionais e pessoais e, consequentemente, diminuem possíveis reações em relação à injustiça percebida.

Neste sentido, a questão de pesquisa que orienta este estudo é a seguinte: As dimensões (controlabilidade e múltiplos indicadores de desempenho) dos Sistemas de Controle Gerencial aumentam a percepção de justiça organizacional e, dessa forma, aumentam o comprometimento e a confiança dos gestores? Assim, o objetivo do estudo é verificar se a percepção de justiça em relação às dimensões dos Sistemas de Controle Gerencial aumenta o comprometimento e a confiança dos gestores.

No Brasil, pesquisas que tratam da justiça organizacional são esparsas, assim, é imperativo o desenvolvimento deste campo de investigação, principalmente por sua utilidade potencial para a melhoria das relações entre trabalhadores e organizações (Assmar, Ferreira, \& Souto, 2005). Estudos sugerem que a justiça percebida em relação às dimensões dos Sistemas de Controle Gerencial afeta significativamente o comportamento de gestores e pode reduzir a propensão de ações retaliatórias (Little, Magner, \& Welker, 2002; Wentzel, 2004).

Little, Magner e Welker (2002) constataram em sua pesquisa que os gestores apresentam um comportamento organizacional particularmente positivo quando percebem os procedimentos orçamentários e a execução desses procedimentos como justos em sua organização. Wentzel (2004) investigou se percepções de equidade diminuem a folga orçamentária em ambientes participativos nos quais os gestores possuem informações privilegiadas. Os resultados sugeriram que a criação de folga orçamentária diminui quando os gestores se consideram justiçados em termos de equidade.

Depreende-se que mais pesquisas são necessárias para ampliar o conhecimento sobre a forma como a justiça organizacional percebida em relação aos SCG pode reduzir o sentimento de injustiça percebida nas empresas, principalmente como o comprometimento e a confiança podem tornar-se fatores chaves nesse processo. A relevância desta pesquisa está em contribuir para o desenvolvimento deste campo de pesquisa na perspectiva contábil, ao abordar aspectos da justiça organizacional sob o enfoque dos SCG. Expande-se, portanto, o campo de estudos organizacionais em contabilidade, ao investigar a relação da justiça organizacional, com dimensões dos SCG e o comprometimento e a confiança dos gestores.

\section{Referencial Teórico}

\section{Justiça organizacional}

Psicólogos sociais têm estudado o que as pessoas pensam estar certo ou errado, ser justo ou injusto e compreender como elas justificam esses julgamentos (Tyler \& Smith, 1998). Correia (2010) afirma que, de fato, é importante conhecer o que o homem comum pensa sobre a justiça, porque a investigação tem mostrado que as pessoas agem e reagem em função do que pensam que é justo. Um ato é apenas justo porque alguém percebe que ele assim o seja (Fortin, 2008).

Justiça é um fenômeno psicossocial e penetra na vida social e organizacional. A literatura sugere que níveis mais elevados de percepções de justiça estão, em geral, associados com atitudes e comportamentos mais positivos no local de trabalho (Moorman, 1991; Rahim, Magner, \& Shapiro, 2000). A justiça organizacional envolve um conjunto de dimensões, a justiça distributiva, a justiça procedimental e a justiça interacional, sendo que esta última pode ser dividida em justiça informativa e justiça interpessoal (Langevin \& Mendoza, 2013).

A justiça distributiva analisa as percepções de justiça dos empregados no que se refere ao que eles recebem da organização, por exemplo, classificações de desempenho, promoções, aumentos salariais, 
atribuição de prêmios de fim de ano e participação em programas de formação. Normalmente, a regra utilizada para estabelecer as comparações entre indivíduos é a regra da equidade, ou seja, reconhecer imparcialmente o direito de cada um (Adams, 1965; Deutsch, 1975; Folger \& Cropanzano, 1998; Homans, 1961; Rego, 2002; Sotomayor, 2006, 2007).

A justiça procedimental estuda as percepções de justiça relativas aos procedimentos que deram origem aos procedimentos organizacionais, por exemplo, os processos de seleção, os sistemas de avaliação de desempenho e os procedimentos de aumentos salariais e promoções (Folger \& Cropanzano, 1998; Greenberg, 1990; Leventhal, 1980; Leventhal et al., 1980; Rego, 2002; Sotomayor, 2007; Thibaut \& Walker, 1975). Há seis regras procedimentais que indicam se um dado procedimento é percebido como justo, ou não, pelos indivíduos. São as denominadas regras procedimentais de Leventhal (1980): consistência, não enviesamento, exatidão da informação, correção (ou mecanismos de correção/recurso), representatividade e eticidade.

A justiça interacional compreende as percepções de justiça relativas ao tratamento interpessoal, isto é, ao tratamento recebido durante e após a implementação dos processos de avaliação. Refere-se ao tratamento respeitoso e cortês por parte dos gestores e às justificativas sobre as decisões tomadas. Segundo Greenberg (1993), a justiça interacional ainda pode ser dividida em informacional e interpessoal. A justiça informacional focaliza-se nas explicações fornecidas às pessoas sobre o porquê de certos procedimentos terem sido utilizados de determinado modo ou o porquê dos resultados terem sido distribuídos de determinada maneira (Colquitt, Conlon, Wesson, Porter, \& Ng, 2001). A justiça interpessoal reflete o grau de cortesia, dignidade e respeito com que as pessoas são tratadas pelas autoridades nos procedimentos ou na determinação de objetivos (Colquitt et al., 2001).

Cada componente da justiça organizacional afeta resultados organizacionais específicos. A justiça distributiva relaciona-se com as percepções de justiça das decisões individuais e afeta resultados pessoais; as percepções de justiça procedimental tendem a afetar atitudes e comportamentos relacionados à organização; e as percepções de justiça interacional (ou a sua partição em justiça interpessoal e informacional) estão mais relacionadas com os comportamentos e atitudes em relação aos decisores (Conner, 2003; Folger \& Cropanzano, 1998; Masterson, Lewis, Goldman, \& Taylor, 2000; Viswesvaran \& Ones, 2002).

Da mesma forma que a percepção de justiça organizacional pode trazer impactos positivos sobre atitudes e comportamentos, a injustiça pode induzir o indivíduo a ter comportamentos negativos. Segundo Leventhal, Karuza e Fry (1980), estudos têm sido realizados no intuito de demonstrar e amenizar os impactos desses comportamentos, sendo que o ambiente de trabalho é um dos locais de convivência social que apresenta maior número de eventos de injustiça, deflagrando vários comportamentos disfuncionais, conforme se pode visualizar na Tabela 1.

Tabela 1

Estudos sobre Injustiça Organizacional

\begin{tabular}{ll}
\hline Autores & Visão \\
\hline Colquitt (2001) & $\begin{array}{l}\text { Injustiças no local de trabalho podem deflagrar processos de estresse, } \\
\text { burnout, perda de comprometimento, absenteísmo e vários outros problemas } \\
\text { de ordem individual e organizacional. }\end{array}$ \\
\hline Cohen-Charash e Spector (2001) & $\begin{array}{l}\text { As injustiças presentes no local de trabalho afetam o comportamento dos } \\
\text { funcionários, além de diminuir sua satisfação, desempenho e piorar os } \\
\text { sentimentos em relação à empresa. Além disso, a saúde do indivíduo pode } \\
\text { ser prejudicada ao criar situações estressantes que, por sua vez, prejudicam o } \\
\text { rendimento da organização. Caso haja a percepção por parte dos funcionários } \\
\text { de que estão sendo vítimas de injustiças, tais indivíduos tendem a responder } \\
\text { negativamente com atos retaliatórios ou adoecimento. }\end{array}$ \\
\hline
\end{tabular}




\section{Tabela 1 (continuação)}

\begin{tabular}{ll}
\hline Autores & Visão \\
\hline Tepper (2001) & A percepção de um ambiente organizacional justo promove atitudes \\
positivas. Os empregados que se percebem tratados com justiça pelos \\
gestores e pela própria organização tendem a acatar as decisões da gerência, \\
apresentar comprometimento socialmente favorável e evitar \\
comportamentos antissociais, além de apresentarem maior \\
comprometimento, qualidade no trabalho, satisfação profissional, saúde, \\
bem-estar e harmonia. \\
\hline Os gestores, por temerem ser tratados de forma injusta, podem acabar criando \\
folga orçamentária e/ou manipular dados como forma alternativa para obter \\
os resultados esperados. Os autores ainda afirmam que um sistema de \\
controle gerencial percebido como injusto irá transmitir a percepção de que \\
os funcionários não são valorizados, o que prejudica o sentimento de \\
identidade com a organização e reduz comportamentos cooperativos.
\end{tabular}

Depreende-se da Tabela 1 que a percepção de injustiça pode acarretar diferentes comportamentos no indivíduo, com reflexos na organização, tais como: estresse, perda de comprometimento, insatisfação profissional e falta de identidade organizacional. Desse modo, os indivíduos tendem a deixar de cumprir os objetivos almejados pela organização para atuar em causa própria, privilegiando seus interesses.

Estudos sobre a percepção de justiça organizacional são recentes, embora o tema justiça organizacional tenha surgido por volta da década de 1960 (Sotomayor, 2007). Em geral, pesquisas mais recentes sobre percepções de justiça organizacional contemplam uma vasta gama de atitudes e comportamentos decorrentes (Beuren \& Santos, 2012; Burney, Henle, \& Widener, 2009; Colquitt, 2001; Filenga \& Siqueira, 2006; Jawahar, 2002; Libby, 1999; Nadiri \& Tanova, 2010; Rego \& Souto, 2004; Seifert, Sweeney, Joireman, \& Thornton, 2010; Sotomayor, 2007; Sousa \& Mendonça, 2009; Zainalipour, Fini, \& Mirkamali, 2010).

\section{Percepção de justiça em relação às dimensões do sistema de controle gerencial}

Sistemas de Controle Gerencial (SCG) são amplamente utilizados nas empresas para assegurar que o comportamento dos empregados tenha congruência com as estratégias e objetivos organizacionais (Merchant \& Van der Stede, 2007). As dimensões incluem todos os mecanismos (planejamento estratégico, orçamento, alocação de recursos, avaliação de desempenho, avaliação e recompensa, centros de responsabilidade e preços de transferência, entre outros) que os gestores podem utilizar para garantir a consistência nas decisões dos subordinados com as estratégias e objetivos da organização (Anthony \& Govindarajan, 2006; Merchant \& Van der Stede, 2007).

Estes mecanismos se consubstanciam no controle gerencial. Segundo Flamholtz (1979), o controle gerencial pode ser visto como o processo de influenciar o comportamento dos membros da organização, aumentando a probabilidade de as pessoas se comportarem de modo a alcançar os objetivos da organização. A motivação dos indivíduos ou grupos é o elemento-chave, não se trata de controlar o comportamento das pessoas de maneira predeterminada, mas influenciá-las a agir em consonância com os objetivos da organização.

Os gestores de uma organização são afetados, quer pelo desenho do SCG e/ou pela sua forma de implementação. Segundo Langevin e Mendoza (2013), o SCG tem impacto sobre a percepção de justiça (ou injustiça) por parte dos gestores. Primeiramente, porque o SCG está intimamente ligado com as questões de justiça distributiva, uma vez que afeta os resultados financeiros. Isso se deve ao fato de que o SCG é usado para determinar os objetivos e a alocação de recursos, e essa última, por sua vez, afeta as oportunidades de obter recompensas. 
O SCG também contém aspectos da justiça processual, uma vez que os procedimentos dependem de como os objetivos são definidos, como o desempenho é avaliado, e as recompensas, concedidas (Langevin \& Mendoza, 2013). Dessa forma, espera-se que esses procedimentos sejam robustos e constantes. Por último, o SCG pode afetar a justiça interacional, considerando que o gestor interage com seus superiores ao negociar suas metas orçamentárias, sua avaliação de desempenho e discute sobre as possíveis recompensas (Langevin \& Mendoza, 2013). A forma como o gestor é tratado nessas interações precisa ser monitorada, porque o gestor pode ser sensível a esse tratamento.

Contemplando apenas um dos elementos do Sistema de Controle Gerencial, no caso, o sistema orçamentário, Little et al. (2002) investigaram o comportamento organizacional dos gestores quando percebem os procedimentos orçamentários como justos em sua organização. Wentzel (2004) investigou a relação da criação da folga orçamentária com as percepções dos gestores quanto à justiça de equidade.

De modo mais abrangente, Langevin e Mendoza (2013) propõem quatro características críticas a serem consideradas no Sistema de Controle Gerencial na perspectiva da justiça organizacional: (a) participação dos gestores na definição de suas metas; (b) uso do princípio da controlabilidade na definição das metas e na avaliação de desempenho; (c) qualidade do feedback; e (d) uso de múltiplas medidas de desempenho. No entanto, essa proposição foi apresentada sob a forma de um construto teórico.

No presente estudo, consideraram-se duas dimensões do Sistema de Controle Gerencial (princípio da controlabilidade e múltiplos indicadores de desempenho), no sentido de verificar se a percepção de justiça em relação a essas dimensões aumenta o comprometimento e a confiança dos gestores. Portanto, avança-se nas pesquisas ao não restringir a pesquisa a um instrumento particular do Sistema de Controle Gerencial, como procedido nos estudos de Little et al. (2002) e Wentzel (2004), além de investigar de forma empírica duas dimensões do modelo teórico de Langevin e Mendoza (2013).

\section{Desenvolvimento das hipóteses}

O princípio da controlabilidade é um dos fatores que deve ser levado em consideração ao estabelecer nas organizações um SCG para fins de avaliação de desempenho dos gestores. Esse princípio é fundamental, se constitui em um pré-requisito para estabelecer um senso de justiça no sistema de avaliação de desempenho (Giraud, Langevin, \& Mendoza, 2008).

A controlabilidade, conforme Aoki (1974, p. 51), "aparece como a técnica necessária ou que oferece condições suficientes para a existência de leis de controle com a propriedade desejada". Segundo Antle e Demski (1988), controlabilidade é definida como "o entendimento de que um gerente deve ser avaliado com base no que ele controla" (p. 700). Para Giraud, Langevin e Mendoza (2008), o princípio da controlabilidade preconiza que "os gerentes devem ser avaliados tendo por base somente elementos que eles possam controlar" (p. 32).

Para Langevin e Mendoza (2013), quando o princípio da controlabilidade não é aplicado, e se eventos incontroláveis afetarem negativamente os resultados, existe o risco de haver um sentimento de que os benefícios concedidos aos gestores não compensaram o esforço por eles despendido. Por outro lado, a aplicação do princípio da controlabilidade irá proteger os gestores da incerteza, além de aumentar a sua percepção de justiça distributiva na relação entre esforço e recompensa.

A aplicação do princípio da controlabilidade, conforme Langevin e Mendoza (2013), é compatível com a maioria das regras procedimentais de Leventhal et al. (1980). Primeiro, a remoção de fatores incontroláveis das avaliações dos gestores aumenta a convicção de que a mensuração do desempenho será relativa aos esforços e não aos acontecimentos imprevisíveis, o que coaduna com a regra de precisão. Segundo, a adoção do princípio da controlabilidade aumenta a coerência dos procedimentos de avaliação de desempenho. Terceiro, sua adoção implica identificar e neutralizar fatores incontroláveis que possam afetar os resultados dos gestores. Quarto, sua adoção cumpre os critérios de eticidade, pois é supostamente mais ético não penalizar os gestores quando seus resultados são afetados negativamente por eventos incontroláveis (Merchant \& Van der Stede, 2007). 
Infere-se do exposto que a aplicação do princípio da controlabilidade influencia positivamente a percepção de justiça distributiva e de justiça processual. Neste sentido, e fundamentado no estudo de Langevin e Mendoza (2013), enunciam-se as seguintes hipóteses:

H1a: A aplicação do princípio da controlabilidade aumenta a justiça distributiva percebida do gestor em relação ao SCG.

H1b: A aplicação do princípio da controlabilidade aumenta a justiça processual percebida do gestor em relação ao SCG.

Além do princípio da controlabilidade, os indicadores financeiros e não-financeiros exigem atenção ao se projetar e implementar um SCG. Segundo Chenhall e Langfield-Smith (2007), os SCG tradicionais contam com diversos indicadores, principalmente financeiros. No entanto, Lau e Moser (2008) e Burney, Henle e Widener (2009) constataram em suas pesquisas que o uso de diversas medidas de desempenho, financeiras e não-financeiras, aumenta a percepção de justiça e, por conseguinte, diminui a tendência de os indivíduos desenvolverem sentimentos de injustiça que possam desencadear ações contrárias aos objetivos organizacionais.

Langevin e Mendoza (2013) destacam que a justiça distributiva e a justiça processual são as mais afetadas por esta dimensão do SCG. A utilização de múltiplas medidas de desempenho afeta fortemente a justiça distributiva, pois aumenta a possibilidade da realização de uma avaliação mais precisa do desempenho. De acordo com Burney et al. (2009), o uso de múltiplas medidas de desempenho facilita a comparação dos indicadores com os resultados e esta comparação é associada com percepções mais elevadas de justiça distributiva dos empregados. A utilização de múltiplas medidas de desempenho também contribui para a justiça processual percebida em relação ao SCG, uma vez que a precisão da informação é um critério fundamental de justiça processual (Leventhal, 1980).

Nesta perspectiva, e fundamentado no estudo de Langevin e Mendoza (2013), para testar a relação entre o uso de múltiplos indicadores de desempenho e as justiças distributiva e processual, enunciam-se as seguintes hipóteses:

H2a: O uso de múltiplos indicadores de desempenho não-financeiros aumenta a justiça distributiva percebida do gestor em relação ao SCG.

H2b: O uso de múltiplos indicadores de desempenho não-financeiros aumenta a justiça processual percebida do gestor em relação ao SCG.

Após a identificação das dimensões do SCG que se presume que possam aumentar a percepção de justiça percebida, faz-se necessário entender como essas dimensões tendem a aumentar o comprometimento e a confiança do gestor e, assim, diminuir a possibilidade de retaliações causadas pelo sentimento de injustiça. Pesquisas revelam que a justiça percebida tem um impacto positivo no comportamento, favorece a satisfação no trabalho, o comprometimento organizacional, a diminuição do estresse, a confiança no superior hierárquico e os comportamentos de cidadania (Cohen-Charash \& Spector, 2001; Colquitt et al., 2001; Viswesvaran \& Ones, 2002).

Para Langevin e Mendoza (2013), o comprometimento organizacional pode ser definido como a identificação e o envolvimento do indivíduo com uma organização em particular. Quando os gestores estão comprometidos com a organização, tendem a se identificar com ela e seus objetivos organizacionais e desejam manter seu vínculo. Portanto, quando os gestores sentem um forte comprometimento organizacional, são menos inclinados a tomar decisões prejudiciais à empresa (Mowday et al., 1979). Nouri (1994) e Nouri e Parker (1996) observaram que um alto nível de comprometimento está fortemente associado com a diminuição do nível de folga orçamentária.

Além do comprometimento, outro efeito positivo da percepção da justiça organizacional é o aumento da confiança. Segundo Rousseau, Sitkin, Burt e Camerer (1998, p. 385), "a confiança é um estado psicológico que compreende a intenção de aceitar a vulnerabilidade baseada em expectativas positivas das intenções ou o comportamento do outro". Para Langevin e Mendoza (2013), a confiança 
nos incentivos transmite maior significado na percepção de controle em relação aos resultados. Para Folger e Greenberg (1985), estas percepções de controle são um componente essencial da justiça organizacional.

Alguns estudos demonstraram que, quando os gestores são recompensados pelo seu desempenho, é mais provável a percepção da equidade ou justiça (Greenberg, 1986, 1990). Outras pesquisas mostraram que todas as dimensões de justiça (distributiva, processual e interacional) estão relacionadas com confiança (Ambrose \& Arnaud, 2005; Cropanzano et al., 2007). Também foi constatado que, se os gestores confiam em seus superiores, eles são menos propensos a desenvolver comportamentos antiéticos, pois a confiança reflete uma expectativa ou crença de que a outra parte vai agir com benevolência (Whitener et al., 1998).

Após um intenso debate sobre quais dimensões da justiça organizacional melhor explicam o comprometimento e a confiança no superior hierárquico, Ambrose e Arnaud (2005) e Cropanzano, Bowen e Gilliland (2007) concluíram em suas pesquisas que as três dimensões aumentam tanto a percepção do comprometimento como a de confiança. Portanto, o pressuposto é que a justiça percebida nos SCG atua em dois níveis: (a) afeta a relação entre o empregado e a organização como um todo (comprometimento organizacional); e (b) influencia as relações dos empregados com seus superiores (confiança no superior).

Com base no exposto, e fundamentado no estudo de Langevin e Mendoza (2013), enunciam-se as seguintes hipóteses:

H3a: A justiça organizacional percebida em relação às dimensões do SCG aumenta o comprometimento do gestor.

H3b: A justiça organizacional percebida em relação às dimensões do SCG aumenta a confiança do gestor no seu superior hierárquico.

Na Figura 1, ilustra-se o modelo formativo com as relações propostas, sendo que o desenho teórico da pesquisa está pautado nas hipóteses enunciadas. De acordo com Chin (1998), indicadores formativos explicam o construto e não se espera correlação entre eles, ao contrário dos indicadores reflexivos, que são explicados pelos construtos.

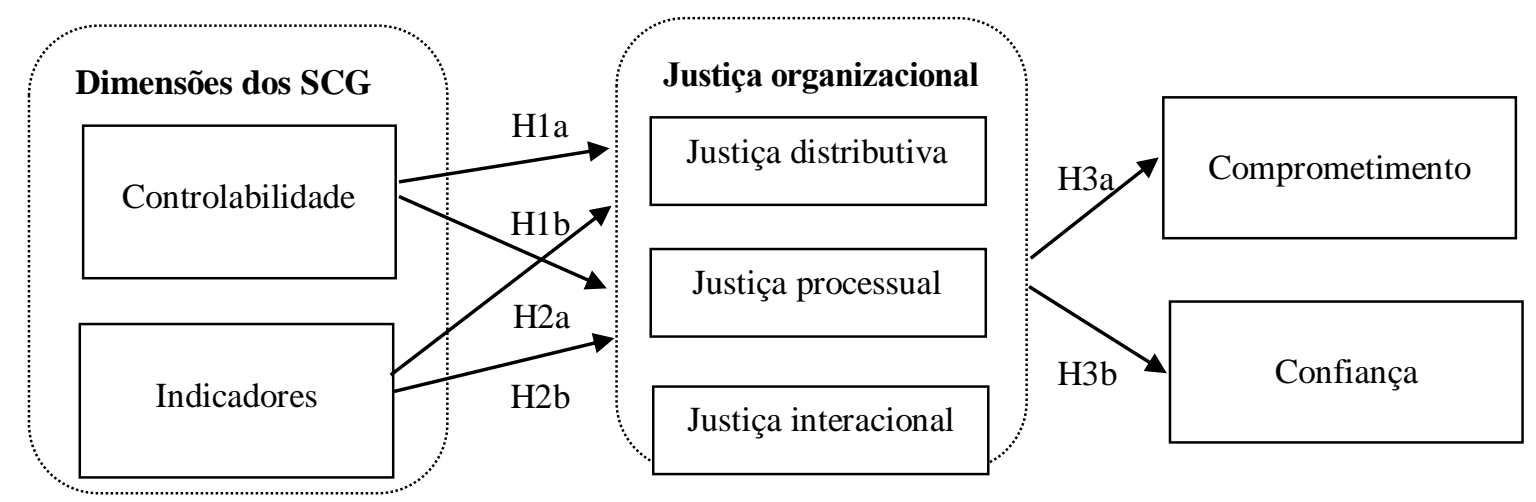

Figura 1. Desenho Teórico da Pesquisa

Depreende-se da Figura 1 que controlabilidade e múltiplos indicadores de desempenho, dimensões dos SCG, aumentam a percepção de justiça organizacional e que essa aumenta o comprometimento e a confiança percebida do gestor. Estas relações de causa e efeito podem se configurar como uma possível solução para a diminuição do sentimento de injustiça percebida pelo gestor. Assim, as relações de causa e efeito podem contribuir para que haja congruência entre os objetivos organizacionais e pessoais, diminuindo a tendência de possíveis ações retaliatórias decorrentes da percepção de injustiça. 


\section{Metodologia da Pesquisa}

Esta pesquisa caracteriza-se como um estudo de natureza descritiva, realizado por meio de pesquisa de levantamento ou survey. A natureza descritiva decorre do propósito da pesquisa, o de verificar se a percepção de justiça em relação às dimensões dos Sistemas de Controle Gerencial aumenta o comprometimento e a confiança dos gestores. A pesquisa de levantamento foi realizada com aplicação de um questionário para uma determinada amostra extraída da população da pesquisa.

A população da pesquisa compreendeu as 500 maiores empresas em faturamento listadas entre as Melhores e Maiores da Revista Exame, edição de 2012. Optou-se por esta população porque grandes empresas tendem a possuir SCG mais consolidados. Para formar a amostra, foram contatadas por telefone as 500 empresas a fim de verificar a sua disponibilidade para responder o questionário e coletar os endereços eletrônicos. Dessas, um total de 180 aceitaram participar da pesquisa, para as quais foi enviado o questionário de forma eletrônica. No entanto, somente 67 retornaram o questionário respondido, representando uma amostra por acessibilidade, o que se constitui em limitação da pesquisa.

O instrumento de pesquisa compõe-se de 26 assertivas formuladas com base no referencial teórico, a fim de testar as hipóteses teóricas contidas no estudo de Langevin e Mendoza (2013), conforme demonstrado na Tabela 2.

Tabela 2

\section{Construtos e Assertivas do Instrumento de Pesquisa}

\begin{tabular}{ll}
\hline Construtos & Assertivas \\
\hline Controlabilidade & $\begin{array}{l}\text { 1. Na definição de metas, são incluídos apenas itens que posso controlar ou em que } \\
\text { posso exercer alguma influência. }\end{array}$ \\
& $\begin{array}{l}\text { 2. Sou recompensado de maneira justa por meus esforços, pois as situações que } \\
\text { posso controlar não são consideradas na minha avaliação. }\end{array}$ \\
& $\begin{array}{l}\text { 3. A minha opinião é considerada nas decisões tomadas em níveis hierárquicos } \\
\text { superiores da empresa. }\end{array}$
\end{tabular}

4. A minha opinião é considerada quando a empresa sofre influência de fatores externos (ambiente econômico e político, decisões de clientes ou concorrentes, entre outros).

Múltiplos 5. No meu trabalho, é levado em consideração o envolvimento e o engajamento das Indicadores de pessoas nos programas institucionais.

Desempenho

6. No meu trabalho, é levado em consideração o nível de conhecimento das pessoas sobre os procedimentos e normas internas necessárias para exercer as atividades.

7. A minha empresa utiliza indicadores não-financeiros formais na avaliação de desempenho de todos os funcionários.

8. No meu trabalho, levam em consideração minha opinião na aplicação de indicadores não-financeiros.

Justiça Distributiva 9. Sou recompensado de maneira justa pela minha experiência profissional, esforços despendidos na realização do meu trabalho e o estresse a que sou submetido em sua execução.

10. Se considerar os demais salários pagos na empresa em que trabalho, recebo um salário justo, e as promoções aos funcionários são realizadas de maneira justa.

11. Na minha empresa, sou recompensado de forma justa pela iniciativa e criatividade nas tomadas de decisões não rotineiras. 


\section{Tabela 2 (continuação)}

\begin{tabular}{|c|c|}
\hline Construtos & Assertivas \\
\hline \multirow[t]{3}{*}{ Justiça Processual } & 12. No meu trabalho, sempre participo da definição dos objetivos da empresa. \\
\hline & $\begin{array}{l}\text { 13. Na minha empresa, recebo feedbacks úteis relativos às decisões que dizem respeito } \\
\text { ao meu trabalho. }\end{array}$ \\
\hline & $\begin{array}{l}\text { 14. Na minha empresa, recebo feedbacks úteis relativos ao meu desempenho (por } \\
\text { exemplo, quais fatores foram considerados, quais os pontos fortes e fracos). }\end{array}$ \\
\hline
\end{tabular}

Justiça Interacional 15. Ao tomar decisões, o meu chefe considera o ponto de vista de todas as pessoas que possam ser afetadas.

16. A minha empresa procura coletar informações precisas antes de tomar as decisões e, se for necessário, os empregados podem recorrer das decisões tomadas.

17. Meus superiores justificam de forma clara, tempestiva e adequada as decisões tomadas na fase de definição de metas.

18. O meu chefe usa o tempo que for necessário para me explicar as decisões tomadas e as suas consequências.

Confiança 19. Considero justos os procedimentos utilizados em minha empresa para definir objetivos e metas, por isso sinto-me mais comprometido com a organização.

20. Na minha empresa, a definição dos objetivos organizacionais é realizada de forma justa, levando em consideração os objetivos pessoais dos funcionários e não apenas da organização.

21. Sou valorizado na minha empresa, pois participo no autocontrole das atividades por mim desenvolvidas.

22. Sou reconhecido na minha empresa, pois nela são considerados apenas fatos concretos e observáveis, não se baseando em suposições ou influências.

Comprometimento 23. Acredito que meu superior hierárquico me represente adequadamente, levando em consideração os meus objetivos na definição das metas organizacionais.

24. Meu superior hierárquico reconhece minha habilidade de administrar prazos e solicitações, e também não realiza demandas excessivas.

25. Meu superior hierárquico sempre me informa sobre minhas habilidades e dificuldades para que eu alcance o nível de desempenho desejado.

26. O meu superior hierárquico fornece justificativas sobre as decisões que são relacionadas ao meu trabalho.

Nota. Adaptado de Giraud, F., Langevin, P., \& Mendoza, C. (2008). Justice as a rationale for the controllability principle: a study of managers' opinions. Management Accounting Research, 19(1), 32-44. doi: 10.1016/j.mar.2007.09.002; Langevin, P., \& Mendoza, C. (2013). How can management control system fairness reduce managers' unethical behaviours? European Management Journal, 31(3), 209-222. doi: 10.1016/j.emj.2012.12.001; Leventhal, G. S. (1980). What should be done with equity theory? New approaches to the study of fairness in social relationships. In K. Gergen, M. Greenberg, \& R. Willis (Orgs.), Social exchange: new advances in theory and research (pp. 27-55). New York: Plenum Press; Merchant, K. A., \& Van der Stede, W. A. (2007). Management control systems: performance measurement, evaluation and incentives (2nd ed.). Edinburgh: Pearson Education; Burney, L. L., Henle, C. A., \& Widener, S. K. (2009). A path model examining the relations among strategic performance measurement system characteristics, organizational justice, and extra-and in-role performance. Accounting, Organizations and Society, 34(3/4), 305-321. doi: 10.1016/j.aos.2008.11.002; Folger, R. G., \& Cropanzano, R. (1998). Organizational justice and human resource management. London: Sage; Rego, A., \& Souto, S. (2004). A percepção de justiça como antecedente do comprometimento organizacional: um estudo luso-brasileiro. Revista de Administração Contemporânea, 8(1), 151-177. doi: 10.1590/S1415-65552004000100008; Colquitt, J. A., Conlon, D. E., Wesson, M. J., Porter, C. O. L. H., \& $\mathrm{Ng}$, K. Y. (2001). Justice at the millennium: a meta-analytic review of 25 years of organizational justice research. Journal of Applied Psychology, 86(3), 425-445. doi: 10.1037//0021-9010.86.3.425; Ambrose, M. L., \& Arnaud, A. (2005). Are procedural justice and distributive justice conceptually distinct? In J. Greenberg \& J. A. Colquitt (Eds.), Handbook of organizational justice (pp. 59-84). Mahwah, NJ: Erlbaum Associates; Cropanzano, R., Bowen, D. E., \& Gilliland, S. W. (2007). The management of organizational justice. Academy of Management Perspectives, 21(4), 34-48. doi: 10.5465/AMP.2007.27895338; Folger, R., \& Greenberg, J. (1985). Procedural justice: an interpretive analysis of personnel systems. In K. Rowland \& G. Ferris (Eds.), Research in personnel and human resources management (pp. 141-183). Greenwich, CT: JAI Press; Cohen-Charash, Y., \& Spector, P. E. (2001). The role of justice in organizations: a meta-analysis. Organizational Behavior and Human Decision Processes, 86(2), 278-321. doi: 10.1006/obhd.2001.2958; e Viswesvaran, C., \& Ones, D. S. (2002). Examining the construct of organizational justice: a meta-analytic evaluation of relations with work attitudes and behaviors. Journal of Business Ethics, 38(3), 193-203. doi: 10.1023/A:1015820708345. 
Observa-se na Tabela 2 que, para cada construto, foram formuladas assertivas, em relação às quais se solicitou aos gestores que indicassem o seu grau de concordância com cada frase em uma escala de sete pontos, sendo: $1=$ discordo totalmente; 2 = discordo moderadamente; $3=$ discordo ligeiramente; 4 $=$ não concordo nem discordo; $5=$ concordo ligeiramente; $6=$ concordo moderadamente; $7=$ concordo totalmente.

Como ferramenta de elaboração e envio do instrumento de pesquisa, foi utilizado o Google docs. O questionário foi enviado aos gestores das empresas que concordaram em participar da pesquisa no período de janeiro a março de 2014, com várias mensagens eletrônicas aos não respondentes para aumentar o tamanho da amostra. Após esse período, os dados coletados foram tabulados em planilha eletrônica e, em seguida, emparelhados para tratamento estatístico descritivo e inferencial.

Para testar as hipóteses, foi utilizada a Modelagem de Equações Estruturais (Structural Equations Modeling [SEM]). Segundo Klem (2006), ela permite testar um conjunto de variáveis, oferecendo a possibilidade de investigar o nível de explicação das variáveis preditoras perante as variáveis dependentes, além de indicar qual a variável preditora é mais importante. Permite ainda determinar se o modelo teórico é válido, perante os dados reais observados (Hox \& Bechger, 1998).

\section{Descrição e Análise dos Resultados}

\section{Perfil dos respondentes}

Na Tabela 3, evidencia-se o perfil dos respondentes da pesquisa, com destaque ao gênero, faixa etária, escolaridade e tempo no cargo ou função na empresa.

Tabela 3

\section{Perfil dos Respondentes}

\begin{tabular}{lcclcc}
\hline Gênero & Frequência & \% & Escolaridade & Frequência & \% \\
\hline Feminino & 23 & 34 & Ensino Médio & 2 & 3 \\
Masculino & 44 & 66 & Superior Incompleto & 6 & 9 \\
& & & Superior Completo & 13 & 19 \\
& & & Especialização & 30 & 45 \\
& & & Mestrado & 16 & 24 \\
\hline Faixa Etária & Frequência & $\mathbf{\%}$ & Tempo no Cargo ou Função & Frequência & \% \\
\hline 25 anos ou menos & 4 & 6 & Missing & 7 & 10 \\
26 a 30 anos & 4 & 6 & Menos que 5 anos & 26 & 39 \\
31 a 35 anos & 16 & 24 & 5 a 10 anos & 23 & 34 \\
36 a 40 anos & 17 & 25 & 11 a 15 anos & 4 & 6 \\
41 a 45 anos & 10 & 15 & 16 a 20 anos & 5 & 8 \\
46 a 50 anos & 5 & 8 & Mais que 20 anos & 2 & 3 \\
51 anos ou mais & 11 & 16 & & & \\
\hline
\end{tabular}

Os dados demográficos identificados na pesquisa, de acordo com a Tabela 3, demonstram que $66 \%$ dos respondentes são do gênero masculino. A maioria (49\%) dos respondentes possui entre 31 a 40 anos. Em relação à escolaridade, observa-se que $88 \%$ dos respondentes possuem nível superior, o 
que denota que existe uma forte preocupação em relação à educação continuada dos participantes da pesquisa.

Com relação ao tempo que o respondente vem exercendo o cargo ou função na empresa, constatou-se que 39\% desempenham a função ou cargo a menos de 5 anos e que $34 \%$ estão desempenhando entre 5 a 10 anos o cargo ou função. Cotejando-se a idade com o tempo na função ou cargo na empresa, depreende-se que os respondentes supostamente tiveram experiências profissionais anteriores em outras empresas.

Os resultados demográficos apresentados sugerem que os respondentes reúnem as condições necessárias para responder o instrumento de pesquisa, em especial considerando-se o seu nível de escolaridade formal.

\section{Equações estruturais (SEM)}

As hipóteses foram analisadas por meio do método de Mínimos Quadrados Parciais (Partial Least Squares Path Modeling [PLS-PM]) para estimação da modelagem de equações estruturais, pelo fato de esse método permitir a modelagem de variáveis latentes com indicadores formativos (Bido, Silva, Souza, \& Godoy, 2010).

A Modelagem de Equações Estruturais, segundo Wold (1982) e Lohmöller (1989), funciona como o sistema de equações simultâneas, mas para a estimativa dos coeficientes com base na análise da estrutura de covariância é feito o ajuste da matriz de covariância, o que implica condições de normalidade e tamanho amostral. Como alternativa, a técnica Partial Least Squares (PLS) é usada para estimar os coeficientes do sistema de equações estruturais com o método dos mínimos quadrados, uma vez que as soluções obtidas são tão confiáveis quanto com a técnica de covariância, e com menos restrições, basicamente na distribuição de dados e tamanho da amostra.

Para usar o PLS, estimou-se o tamanho necessário da amostra, utilizando o G*Power (Faul, Erdfelder, Buchner, \& Lang, 2009); no caso, avaliou-se a variável latente que recebe o maior número de setas. No cálculo, deve-se observar que há dois parâmetros: o poder do teste (Power $=1-\beta$ erro prob. II) e o tamanho do efeito (f2). Hair, Anderson, Tatham e Black (2005) recomendam para o uso do poder de teste de 0,80 um efeito mediano de 0,15. Considerando os três preditores do modelo estrutural apresentado na Figura 1, com uma probabilidade de erro de 10\%, a amostra a ser investigada é de 62 casos. Portanto, a amostra de 67 casos desta pesquisa se mostra adequada para a análise das hipóteses por meio do PLS, via bootstrapping.

O bootstrap calcula os intervalos de confiança dos parâmetros em circunstâncias nas quais outras técnicas não são aplicáveis, em particular quando o número de elementos da amostra é reduzido. De modo geral, o bootstrapping tende a gerar coeficientes de caminhos mais estáveis do que o jackknifing e, portanto, $p$ values mais confiáveis para amostras pequenas (menores que 100 observações) (Chiquoine \& Hjalmarsson, 2009).

O uso do bootstrap, ao invés do tradicional teste $t$, permite verificar a significância de estimativas de parâmetros que não partem da premissa de normalidade multivariada (Azevedo et al., 2012). Optouse por rodar o modelo com o máximo de repetições possíveis (999).

Para executar o modelo de equações estruturais, primeiro procedeu-se à validação dos construtos por meio da análise fatorial nos indicadores, realizada pelo método de extração denominado análise de componentes principais, e método de rotação Varimax com normalização Kaiser. Com esse procedimento seria necessário excluir as variáveis pertencentes aos construtos de justiça organizacional (distributiva, processual e interacional), contudo, optou-se por mantê-las no modelo a fim de analisar se as variáveis latentes se mantêm no contexto da análise.

Após isso, avaliou-se o viés decorrente do método comum de coleta de dados (Common Method Bias [CMB]), por meio da metodologia proposta por Podsakoff, MacKenzie, Lee e Podsakoff (2003) e 
Williams, Edwards e Vandenberg (2003), e utilizada por Liang, Saraf, Hu e Xue (2007) para a estimação via Partial Least Squares (PLS). O viés pode causar correlação espúria entre indicadores ou construtos em função da forma comum de coleta de dados dos indicadores. Podsakoff e Organ (1986) explicam que a correlação espúria ocorre quando indicadores que não deveriam estar correlacionados apresentam correlação em decorrência da utilização do mesmo método de coleta de dados. Assim, recomendam a inclusão de uma variável latente no modelo, que represente o método de coleta de dados.

Os resultados da avaliação do viés sugerem uma forte correlação entre a variável inserida e as variáveis latentes do modelo. Portanto, os resultados a seguir apresentados foram analisados após o controle do viés. Primeiramente, avaliou-se o modelo de mensuração, por meio do alfa de Cronbach e da variância média explicada, conforme Tabela 4. Ambos têm a função de avaliar se o indicador mensurou adequadamente os construtos (Fornell \& Larcker, 1981; Hair, Anderson, Tatham, \& Black, 2005). A avaliação pelo alfa de Cronbach consiste em calcular a correlação que existe entre cada item do teste e o restante dos itens ou o total dos itens (Pasquali, 2001). Segundo Hair et al. (2005), o alfa de Cronbach estabelece a expectativa de erro da medida, sendo que, quanto mais próximo de 1,00, menor a expectativa de erro e maior a confiabilidade do instrumento.

Tabela 4

\section{Índices de Adequação do Modelo SEM-PLS}

\begin{tabular}{lcccc}
\hline Construtos & AVE & Alfa de Cronbach & R Square & $\begin{array}{c}\text { Confiabilidade } \\
\text { Composta }\end{array}$ \\
\hline Comprometimento & 0,8225 & 0,9277 & 0,7856 & 0,9488 \\
Confiança & 0,7355 & 0,8795 & 0,8815 & 0,9173 \\
Controlabilidade & 0,6204 & 0,7950 & 0,6436 & 0,8671 \\
Indicadores & 0,5826 & 0,7635 & 0,7590 & 0,8474 \\
Justiça Distributiva & 0,7066 & 0,7955 & 0,6311 & 0,8784 \\
Justiça Interacional & 0,5651 & 0,7396 & 0,5752 & 0,8376 \\
Justiça Processual & 0,6794 & 0,7500 & 0,7405 & 0,8616 \\
\hline
\end{tabular}

De acordo com a Tabela 4, os resultados do alfa de Cronbach foram superiores a 0,70, demonstrando um grau de fidelidade aceitável (Hair et al., 2005). Portanto, aceitam-se os indicadores para cada construto, sendo que a menor carga foi de 0,7396 para justiça interacional. Esse resultado condiz com os estudos de Colquitt (2001) na validação do modelo conceitual de justiça organizacional, composto de três fatores (justiça distributiva, processual e interacional). O modelo de três fatores é a conceituação mais comumente usada para avaliar a justiça organizacional, o qual também foi validado por Aryee, Budhwar e Chen (2002).

A Variância Média Explicada (Average Variance Extracted [AVE]) representa a variação média compartilhada entre um construto e suas variáveis (Fornell \& Larcker, 1981). Para a validade convergente, a AVE deve ser superior a 0,50 (Hair et al., 2005). Na Tabela 4, observa-se que os resultados foram superiores a 0,50 (Hair et al., 2005) para todas as variáveis latentes. Isso indica a validade convergente e reflete a quantia geral de variância nos indicadores explicada pelo construto latente. Como os indicadores representam o construto latente, não se fez necessário excluir indicadores com cargas menores.

Outro aspecto verificado é a validade discriminante. Para tal, Fornell e Larcker (1981) recomendam a comparação da AVE de cada construto com as variâncias compartilhadas entre os construtos, ou seja, os valores da variância compartilhada devem ser menores do que o da AVE na maioria dos construtos. Para os construtos estudados, foi verificada a validade discriminante entre todas as dimensões, conforme pode ser observado na Tabela 5, em que a AVE (destacada na diagonal em negrito) é maior do que a variância compartilhada entre as dimensões. 
Tabela 5

Validade Discriminante

\begin{tabular}{lccccccc}
\cline { 2 - 7 } & Compr. & Conf. & Control. & Indic. & J. distrib. & J. interac. J. processual \\
\hline Comprometimento & $\mathbf{0 , 9 4 8 8}$ & & & & & & \\
Confiança & 0,7239 & $\mathbf{0 , 9 1 7 3}$ & & & & & \\
Controlabilidade & 0,4633 & 0,7252 & $\mathbf{0 , 8 6 7 1}$ & & & & \\
Indicadores & 0,7380 & 0,7975 & 0,5928 & $\mathbf{0 , 8 4 7 4}$ & & & \\
Justiça distributiva & 0,4370 & 0,7227 & 0,6618 & 0,7046 & $\mathbf{0 , 8 7 8 4}$ & & \\
Justiça interacional & 0,5725 & 0,6097 & 0,6065 & 0,5423 & 0,5839 & $\mathbf{0 , 8 3 7 6}$ & \\
Justiça processual & 0,6070 & 0,7523 & 0,7065 & 0,6848 & 0,6882 & 0,6198 & $\mathbf{0 , 8 6 1 6}$ \\
\hline
\end{tabular}

A terceira etapa, desenvolvida conforme orientação de Hair et al. (2005), foi a validação do modelo estrutural. Esta consiste da verificação dos valores do $\mathrm{R}^{2}$, que corresponde à porcentagem de variância de uma variável latente explicada por outras variáveis latentes. Os valores do $\mathrm{R}^{2}$ fornecem uma medida relativa de ajuste para cada equação estrutural, e apenas são fornecidos para variáveis latentes endógenas. A Tabela 4 indica que o menor $R^{2}$ entre os construtos foi de 0,5752 para a variável latente justiça interacional.

Outro aspecto observado foi o teste t para o caminho (path) utilizado no modelo, sendo aceitáveis aqueles acima de 1,96, conforme Hair et al. (2005). A análise de relação é demonstrada na Tabela 6.

Tabela 6

\section{Resultados do PLS - Path Coeficiente}

\begin{tabular}{lcccc}
\hline Relação Estrutural & Valor & t-valor & Hipótese & p-valor \\
\hline Controlabilidade/Justiça distributiva & 0,0456 & 0,2775 & $\mathrm{H} 1 \mathrm{a}$ & 0,7814 \\
Controlabilidade/Justiça processual & $-0,0483$ & 0,3956 & $\mathrm{H} 1 \mathrm{~b}$ & 0,6925 \\
Indicadores/Justiça distributiva & 0,0270 & 0,1331 & $\mathrm{H} 2 \mathrm{a}$ & 0,8941 \\
Indicadores/Justiça processual & $-0,0293$ & 1,7285 & $\mathrm{H} 2 \mathrm{~b}$ & 0,0842 \\
Justiça distributiva/Comprometimento & $-0,6067$ & 4,5866 & $\mathrm{H} 3 \mathrm{a}$ & $0,0000^{* *}$ \\
Justiça interacional/Comprometimento & $-0,0855$ & 0,9908 & $\mathrm{H} 3 \mathrm{a}$ & 0,3220 \\
Justiça processual/Comprometimento & $-0,1629$ & 1,4924 & $\mathrm{H} 3 \mathrm{a}$ & 0,1359 \\
Justiça distributiva/Confiança & $-0,2512$ & 3,0129 & $\mathrm{H} 3 \mathrm{~b}$ & $0,0027^{* *}$ \\
Justiça interacional/Confiança & $-0,3261$ & 2,0181 & $\mathrm{H} 3 \mathrm{~b}$ & $0,0439^{*}$ \\
Justiça processual/Confiança & $-0,1869$ & 1,8987 & $\mathrm{H} 3 \mathrm{~b}$ & 0,0579 \\
\hline
\end{tabular}

Nota. * Significante $\mathrm{p}<0,05 / * *$ significante $\mathrm{p}<0,01$.

Observa-se, na Tabela 6, que apenas a relação entre as variáveis latentes justiça distributiva e comprometimento, justiça distributiva e confiança, justiça interacional e confiança apresentaram valores acima de 1,96 para os coeficientes de caminho.

Os resultados observados na Tabela 6 permitem analisar as hipóteses enunciadas nesta pesquisa. A primeira hipótese (H1a e H1b) buscou verificar se a aplicação do princípio da controlabilidade está significativamente relacionada com a justiça distributiva e a justiça processual. Os resultados não foram significativos, ou seja, a aplicação do princípio da controlabilidade não influencia a percepção dos 
gestores de considerar o SCG mais justo. Contrariando a proposição de Langevin e Mendoza (2013), que ao respeitar o princípio da controlabilidade, a organização protege seus gestores da incerteza e aumenta a percepção de justiça.

A segunda hipótese (H2a e H2b) testou a relação entre a utilização de múltiplos indicadores de desempenho e a justiça distributiva e processual. Os resultados não foram significativos, ou seja, a utilização de múltiplas medidas de desempenho não afeta a percepção de justiça distributiva e processual. Assim, considerando os preceitos teóricos, pode-se inferir, para esta amostra, que os gestores não presumem que a utilização de múltiplos indicadores aumenta a possibilidade de uma avaliação mais precisa do seu real desempenho, resultado este que contraria o observado nos estudos de Leventhal (1980), Lau e Moser (2008) e Burney et al. (2009).

A terceira hipótese (H3a) de pesquisa visa testar se a percepção de justiça organizacional aumenta o comprometimento organizacional. Considerando que a justiça organizacional é tridimensional, ou seja, que engloba os componentes distributivos, processuais e interacionais, os resultados foram significativos a um $\mathrm{p}<0,01$ para a dimensão distributiva. As dimensões processual e interacional não apresentaram significância. Este resultado difere do observado no estudo de Ambrose e Arnaud (2005) e Cropanzano et al. (2007), que constaram que as três dimensões da justiça organizacional aumentam a percepção do comprometimento. Em relação a hipótese $\mathrm{H} 3 \mathrm{~b}$, que visa verificar se a justiça organizacional percebida aumenta a confiança no superior hierárquico, os resultados foram significativos a um $\mathrm{p}<0,01$ para a dimensão distributiva, e a um $\mathrm{p}>0,05$ para a dimensão interacional. A dimensão processual não apresentou significância, resultado que difere dos achados de Ambrose e Arnaud (2005) e Cropanzano et al. (2007).

\section{Conclusões}

O estudo objetivou investigar se a percepção de justiça em relação às dimensões dos Sistemas de Controle Gerencial aumenta o comprometimento e a confiança dos gestores. Para tal, uma pesquisa descritiva foi realizada a partir de um levantamento junto às 500 empresas listadas entre as Melhores e Maiores da Revista Exame, das quais 180 aceitaram participar do estudo, e a amostra por acessibilidade compreendeu os 67 respondentes. O instrumento de pesquisa utilizado no levantamento compõe-se de 26 assertivas, com escalas de medidas intervalares Likert de sete pontos, agrupadas em cinco blocos: controlabilidade, múltiplos indicadores de desempenho, justiça organizacional, comprometimento e confiança. Além disso, no final do questionário foram solicitados alguns dados demográficos dos respondentes. $\mathrm{Na}$ análise dos dados, utilizou-se estatística descritiva e inferencial e, para testar as hipóteses, a Modelagem de Equações Estruturais (SEM).

Os dados demográficos dos respondentes da pesquisa indicaram que a maioria é do gênero masculino e situa-se na faixa etária até 40 anos. O tempo na função ou cargo na empresa que prevaleceu foi entre 5 a 10 anos, o que sugere experiência anterior em outras empresas. Um aspecto que chamou a atenção é o elevado nível de escolaridade dos respondentes da pesquisa, sendo que mais da metade possui curso de pós-graduação lato sensu ou stricto sensu, em nível de especialização ou de mestrado, o que denota que existe uma forte preocupação em relação à educação continuada. Essas características podem ser um indicativo de que eles reúnem as condições para responder o instrumento de pesquisa.

Os resultados da pesquisa indicaram que o modelo teórico proposto se mostra adequado aos objetivos do estudo. No teste das hipóteses, em relação aos dois primeiros conjuntos de hipóteses testados, os resultados não foram condizentes com o observado na revisão da literatura, ou seja, a aplicação do princípio da controlabilidade não influencia a percepção dos gestores a considerar o SCG mais justo (H1a e H1b), e a utilização de múltiplas medidas de desempenho não afeta a percepção de justiça distributiva e de justiça processual (H2a e H2b). 
A hipótese $\mathrm{H} 3 \mathrm{a}$ foi parcialmente aceita, pois os resultados indicaram que apenas a justiça distributiva influencia o comprometimento dos gestores, o que difere dos resultados de Ambrose e Arnaud (2005) e Cropanzano et al. (2007), em que as três dimensões da justiça organizacional levam ao aumento do comprometimento. Da mesma forma, a hipótese $\mathrm{H} 3 \mathrm{~b}$ foi parcialmente aceita, nesse caso, as dimensões da justiça que influenciam na confiança são a justiça interacional e a distributiva, cujo resultado também difere de Ambrose e Arnaud (2005) e Cropanzano et al. (2007).

Em síntese, os resultados da pesquisa demonstram que o princípio da controlabilidade e a utilização de múltiplos indicadores de desempenho não aumentam a percepção de justiça distributiva e de justiça processual; a percepção de justiça da dimensão distributiva aumenta o comprometimento dos gestores; e a confiança dos gestores é influenciada pela percepção de justiça distributiva e interacional.

Portanto, conclui-se que o comprometimento e a confiança podem ser fatores atitudinais e comportamentais preponderantes na diminuição de ações retaliatórias causadas pelo sentimento de injustiça. No entanto, esta inferência precisa ser matizada, pois outras dimensões do SCG podem influenciar na percepção de justiça organizacional, podendo revelar outras atitudes e comportamentos que contribuam para aumentar o sentimento de justiça e diminuir ações que possam indiretamente prejudicar a organização.

Os resultados do estudo instigam novas pesquisas, especialmente em função das estratégias metodológicas impostas ao seu desenvolvimento. Destaca-se que os dados usados na análise PLS foram coletados de apenas uma fonte, o que acarretou no viés do método comum. Assim, recomenda-se que pesquisas futuras coletem os dados de fontes independentes. Recomenda-se ainda que futuras pesquisas verifiquem de que forma outras dimensões dos SCG, como definição de metas e feedback, podem mitigar atitudes e comportamentos incongruentes com os objetivos organizacionais.

\section{Referências}

Adams, J. S. (1965). Inequity in social exchange. In L. Berkowitz (Ed.), Advances in experimental social psychology (pp. 267-299). New York: Academic Press.

Ambrose, M. L., \& Arnaud, A. (2005). Are procedural justice and distributive justice conceptually distinct? In J. Greenberg \& J. A. Colquitt (Eds.), Handbook of organizational justice (pp. 59-84). Mahwah, NJ: Erlbaum Associates.

Anthony, R. N., \& Govindarajan, V. (2006). Sistemas de controle gerencial. São Paulo: Atlas.

Antle, R., \& Demski, J. S. (1988). The controllability principle in responsibility accounting. Accounting Review, 63(1), 700-718.

Aoki, M. (1974). Local controllability of a decentralizes economic system. The Review of Economic Studies, 41(1), 51-63. doi: 10.2307/2296398

Aryee, S., Budhwar, P. S., \& Chen, Z. X. (2002). Trust as a mediator of the relationship between organizational justice and work outcomes: test of a social exchange model. Journal of Organizational Behavior, 23(3), 267-285. doi: 10.1002/job.138

Assmar, E. M. L., Ferreira, M. C., \& Souto, S. O. (2005). Justiça organizacional: uma revisão crítica da literatura. Psicologia: Reflexão e Crítica, 18(3), 443-453. doi: 10.1590/S010279722005000300019

Azevedo, C. E. F. A., Oliveira, L. G. L., Abdalla, M. M., Gonzalez, R. K., Ribeiro, A. J. G., \& Holperin, M. M. (2012). Por que Finanças? Avaliando o interesse dos estudantes de graduação em administração pela área de finanças. Revista de Administração Mackenzie, 13(6), 168-196. 
Beuren, I. M., \& Santos, V. (2012). Percepção de justiça organizacional na avaliação de desempenho de controllers. Enfoque: Reflexão Contábil, 31(3), 53-72. doi: 10.4025/enfoque.v31i3.16863

Bido, D. S., Silva, D., Souza, C. A., \& Godoy, A. S. (2010). Mensuração com indicadores formativos nas pesquisas em administração de empresas: como lidar com a multicolinearidade entre eles? Administração: Ensino e Pesquisa, 11(2), 245-269. doi: 10.13058/raep.2010.v11n2.145

Burney, L. L., Henle, C. A., \& Widener, S. K. (2009). A path model examining the relations among strategic performance measurement system characteristics, organizational justice, and extra-and in-role performance. Accounting, Organizations and Society, 34(3/4), 305-321. doi: 10.1016/j.aos.2008.11.002

Chenhall, R. H., \& Langfield-Smith, K. (2007). Multiple perspectives of performance measures. European Management Journal, 25(4), 266-282. doi: 10.1016/j.emj.2007.06.001

Chin, W. W. (1998). The partial least squares approach for structural equation modeling. In G. A. Marcoulides (Ed.), Modern methods for business research (pp. 295-336). New Jersey: Lawrence Erlbaum Associates.

Chiquoine, B., \& Hjalmarsson, E. (2009). Jackknifing stock return predictions. Journal of Empirical Finance, 16(5), 793-803. doi: 10.1016/j.jempfin.2009.07.003

Cohen-Charash, Y., \& Spector, P. E. (2001). The role of justice in organizations: a meta-analysis. Organizational Behavior and Human Decision Processes, 86(2), 278-321. doi: 10.1006/obhd.2001.2958

Colquitt, J. A. (2001). On the dimensionality of organizational justice: a construct validation of a measure. Journal of Applied Psychology, 86(3), 386-400. doi: 10.1037//0021-9010.86.3.386

Colquitt, J. A., Conlon, D. E., Wesson, M. J., Porter, C. O. L. H., \& Ng, K. Y. (2001). Justice at the millennium: a meta-analytic review of 25 years of organizational justice research. Journal of Applied Psychology, 86(3), 425-445. doi: 10.1037//0021-9010.86.3.425

Conner, D. S. (2003). Socially appraising justice: a cross-cultural perspective. Social Justice Research, 16(1), 29-39. doi: 10.1023/A:1022922010184

Correia, I. (2010). Psicologia social da justiça: fundamentos e desenvolvimentos teóricos e empíricos. Análise Psicológica, 28(1), 7-28.

Cropanzano, R., Bowen, D. E., \& Gilliland, S. W. (2007). The management of organizational justice. Academy of Management Perspectives, 21(4), 34-48. doi: 10.5465/AMP.2007.27895338

Cropanzano, R., \& Folger, R. (1989). Referent cognitions and task decision autonomy: beyond equity theory. Journal of Applied Psychology, 74(2), 293-299. doi: 10.1037/0021-9010.74.2.293

Defond, M. L., \& Park, C. W. (1997). Smoothing income in anticipation of future earnings. Journal of Accounting and Economics, 23(2), 115-139. doi: 10.1016/S0165-4101(97)00004-9

Deutsch, M. (1975). Equity, equality, and need: what determines which value will be used as the basis for distributive justice? Journal of Social Issues, 31(3), 137-149. doi: 10.1111/j.15404560.1975.tb01000.x

Dunk, A. S. (1993). The effect of budget emphasis and information asymmetry on the relation between budgetary participation and slack. The Accounting Review, 68(2), 400-410.

Faul, F., Erdfelder, E., Buchner, A., \& Lang, A.-G. (2009). Statistical power analyses using G*Power 3.1: tests for correlation and regression analyses. Behavior Research Methods, 41(4), 1149-1160. doi: 10.3758/BRM.41.4.1149 
Filenga, D., \& Siqueira, M. M. M. (2006). O impacto de percepções de justiça em três bases de comprometimento organizacional. Revista de Administração da USP, 41(4), 431-441.

Flamholtz, E. (1979). Organizational control systems as a managerial tool. California Management Review, 22(2), 50-59. doi: 10.2307/41165320

Folger, R. G., \& Cropanzano, R. (1998). Organizational justice and human resource management. London: Sage.

Folger, R., \& Greenberg, J. (1985). Procedural justice: an interpretive analysis of personnel systems. In K. Rowland \& G. Ferris (Eds.), Research in personnel and human resources management (pp. 141-183). Greenwich, CT: JAI Press.

Fornell, C., \& Larcker, D. F. (1981). Evaluating structural equation models with unobservable variable sand measurement error. Journal of Marketing Research, 18(1), 39-50. doi: 10.2307/3151312

Fortin, M. (2008). Perspectives on organizational justice: concept clarification, social context integration, time and links with morality. International Journal of Management Reviews, 10(2), 93-126. doi: 10.1111/j.1468-2370.2008.00231.x

Giraud, F., Langevin, P., \& Mendoza, C. (2008). Justice as a rationale for the controllability principle: a study of managers' opinions. Management Accounting Research, 19(1), 32-44. doi: 10.1016/j.mar.2007.09.002

Greenberg, J. (1986). Determinants of perceived fairness of performance evaluations. Journal of Applied Psychology, 71(2), 340-342.

Greenberg, J. (1990). Organizational justice: yesterday, today and tomorrow. Journal of Management, 16(2), 399-432. doi: 10.1177/014920639001600208

Greenberg, J. (1993). The intellectual adolescence of organizational justice: you've come a long way, maybe. Social Justice Research, 6(1), 135-148. doi: 10.1007/BF01048736

Hair, J. F., Jr., Anderson, R. E., Tatham, R. L., \& Black, W. C. (2005). Análise multivariada de dados. Porto Alegre: Bookman.

Homans, G. C. (1961). Social behavior: its elementary forms. London: Routledge and Kegan Paul.

Hox, J. J., \& Bechger, T. M. (1998). An introduction to structural equation modeling. Family Science Review, 11(1), 354-373.

Jawahar, I. M. (2002). A model of organizational justice and workplace aggression. Journal of Management, 28(6), 811-834. doi: 10.1016/S0149-2063(02)00189-7

Klem, L. (2006). Structural equation modeling. In L. G. Grimm \& P. R. Yarnold (Eds.), Reading and understanding more multivariate statistics (pp. 227-260). Washington: American Psychological Association.

Langevin, P., \& Mendoza, C. (2013). How can management control system fairness reduce managers' unethical behaviours? European Management Journal, 31(3), 209-222. doi: 10.1016/j.emj.2012.12.001

Lau, C. M., \& Moser, A. (2008). Behavioral effects of nonfinancial performance measures: the role of procedural fairness. Behavioral Research in Accounting, 20(2), 55-71. doi: 10.2308/bria.2008.20.2.55 
Leventhal, G. S. (1980). What should be done with equity theory? New approaches to the study of fairness in social relationships. In K. Gergen, M. Greenberg, \& R. Willis (Orgs.), Social exchange: new advances in theory and research (pp. 27-55). New York: Plenum Press.

Leventhal, G. S., Karuza, J., \& Fry, W. R. (1980). Beyond fairness: a theory of allocation preferences. In G. Mikula (Ed.), Justice and social interaction: experimental and theorical contributions from psychological research (pp. 167-218). New Yourk: Springer-Verlag.

Liang, H., Saraf, N., Hu, Q., \& Xue, Y. (2007). Assimilation of enterprise systems: the effect of institutional pressures and the mediating role of top management. MIS Quartely, 31(1), 59-87. doi: $10.2307 / 25148781$

Libby, T. (1999). The influence of voice and explanation on performance in a participative budgeting setting. Accounting, Organizations and Society, 24(2), 125-137. doi: 10.1016/S03613682(98)00043-9

Little, H. T., Magner, N. R., \& Welker, R. B. (2002). The fairness of formal budgetary procedures and their enactment relationships with managers' behavior. Group \& Organization Management, 27(2), 209-225. doi: 10.1177/10501102027002003

Lohmöller, J.-B. (1989). Latent variable path modeling with partial least squares. Heidelberger: Physica-Verlag.

Masterson, S. S., Lewis, K., Goldman, B. M., \& Taylor, M. S. (2000). Integrating justice and social exchange: the differing effects of fair procedures and treatment on work relationships. Academy of Management Journal, 43(4), 738-748. doi: 10.2307/1556364

Merchant, K. A., \& Rockness, J. (1994). The ethics of managing earnings: an empirical investigation. Journal of Accounting and Public Policy, 13(1), 79-94. doi: 10.1016/0278-4254(94)90013-2

Merchant, K. A., \& Van der Stede, W. A. (2007). Management control systems: performance measurement, evaluation and incentives (2nd ed.). Edinburgh: Pearson Education.

Moorman, R. H. (1991). Relationship between organizational justice and organizational citizenship behaviors: do fairness perceptions influence employee citizenship? Journal of Applied Psychology, 76(6), 845-855. doi: 10.1037/0021-9010.76.6.845

Mowday, R. T., Steers, R. M., \& Porter, L. W. (1979). The measurement of organizational commitment. Journal of Vocational Behavior, 14(2), 224-247. doi: 10.1016/0001-8791(79)90072-1

Nadiri, H., \& Tanova, C. (2010). An investigation of the role of justice in turnover intentions, job satisfaction, and organizational citizenship behavior in hospitality industry. International Journal of Hospitality Management, 29(1), 33-41. doi: 10.1016/j.ijhm.2009.05.001

Nouri, H. (1994). Using organizational commitment and job involvement to predict budgetary slack a research note. Accounting, Organizations \& Society, 19(3), 289-295.

Nouri, H., \& Parker, R. J. (1996). The effect of organizational commitment on the relation between budgetary participation and budgetary slack. Behavioral Research in Accounting, 8(1), 74-90.

Omar, A. (2006). Justicia organizacional, individualismo-colectivismo y estrés laboral. Psicologia y Salud, 16(2), 207-217.

Parker, R. J., \& Kohlmeyer, J. M., III (2005). Organizational justice and turnover in public accounting firms: a research note. Accounting, Organizations and Society, 30(4), 357-369. doi: 10.1016/j.aos.2004.05.001

Pasquali, L. (2001). Técnicas de exame psicológico. São Paulo: Casa do Psicólogo. 
Podsakoff, P. M., MacKenzie, S. B., Lee, J.-Y., \& Podsakoff, N. P. (2003). Common method biases in behavioral research: a critical review of the literature and recommended remedies. Journal of Applied Psychology, 88(5), 879-903. doi: 10.1037/0021-9010.88.5.879

Podsakoff, P. M., \& Organ, D. W. (1986). Self-reports in organizational research: problems and prospects. Journal of Management, 12(4), 531-544. doi: 10.1177/014920638601200408

Rahim, M. A., Magner, N. R., \& Shapiro, D. L. (2000). Do justice perceptions influence styles of handling conflict with supervisors?: what justice perceptions, precisely?. International Journal of Conflict Management, 11(1), 9-31. doi: 10.1108/eb022833

Rego, A. (2002). Comprometimento afectivo dos membros organizacionais: o papel das percepções de justiça. Revista de Administração Contemporânea, 6(2), 209-241. doi: 10.1590/S141565552002000200012

Rego, A., \& Souto, S. (2004). A percepção de justiça como antecedente do comprometimento organizacional: um estudo luso-brasileiro. Revista de Administração Contemporânea, 8(1), 151177. doi: 10.1590/S1415-65552004000100008

Rousseau, D. M., Sitkin, S. B., Burt, R. S., \& Camerer, C. (1998). Not so different after all: a crossdiscipline view of trust. Academy of Management Review, 23(3), 393-404. doi: 10.5465/AMR.1998.926617

Seifert, D. L., Sweeney, J. T., Joireman, J., \& Thornton, J. M. (2010). The influence of organizational justice on accountant whistleblowing. Accounting, Organizations and Society, 35(7), 707-717. doi: 10.1016/j.aos.2010.09.002

Skarlicki, D. P., Folger, R., \& Tesluk, P. (1999). Personality as a moderator in the relationship between fairness and retaliation. Academy of Management Journal, 42(1), 100-108. doi: 10.2307/256877

Sotomayor, A. M. S. B. (2006). As percepções de justiça organizacional na avaliação de desempenho: consequências sobre o compromisso organizacional e o compromisso face ao superior hierárquico (Tese de doutorado). Instituto Superior de Economia e Gestão, Universidade Técnica de Lisboa, Lisboa, Portugal.

Sotomayor, A. M. S. B. (2007). Avaliação de desempenho e compromisso organizacional: a perspectiva da justiça organizacional. Revista Universo Contábil, 3(3), 87-100.

Sousa, I. A. C. M. O., \& Mendonça, H. (2009). Justiça organizacional, prazer e sofrimento no trabalho: análise de um modelo mediacional. Revista de Administração Mackenzie, 10(4), 57-74. doi: $10.1590 / \mathrm{S} 1678-69712009000400004$

Tepper, B. J. (2001). Health consequences of organizational injustice: tests of main and interactive effects. Organizational Behavior and Human Decision Processes, 86(1), 197-215. doi: 10.1006/obhd.2001.2951

Thibaut, J., \& Walker, L. (1975). Procedural justice: a psychological analysis. Hillsdale, NJ: Erlbaum.

Tyler, T. R., \& Smith, H. J. (1998). Social justice and social movements [Working Paper $\mathrm{n}^{\mathbf{o}}$ 61]. University of California, Berkeley, CA, EUA.

Viswesvaran, C., \& Ones, D. S. (2002). Examining the construct of organizational justice: a metaanalytic evaluation of relations with work attitudes and behaviors. Journal of Business Ethics, 38(3), 193-203. doi: 10.1023/A:1015820708345

Wentzel, K. (2004). Do perceptions of fairness mitigate managers use of budgetary slack during asymmetric information conditions? Advances in Management Accounting, 13(1), 223-244. doi: 10.1016/S1474-7871(04)13009-8 
Whitener, E. M., Brodt, S. E., Korsgaard, M. A., \& Werner, J. M. (1998). Managers as initiators of trust: an exchange relationship framework for understanding managerial trustworthy behavior. Academy of Management Review, 23(3), 513-530. doi: 10.2307/259292

Williams, L. J., Edwards, J. R., \& Vandenberg, R. J. (2003). Recent advances in causal modeling methods for organizational and management research. Journal of Management, 29(6), 903-936. doi: 10.1016/S0149-2063_03_00084-9

Wold, H. (1982). Systems under indirect observation. In C. Fornell (Ed.), A second generation of multivariate analysis (pp. 325-347). New York: Praeger Publishers.

Zainalipour, H., Fini, A. A. S., \& Mirkamali, S. M. (2010). A study of relationship between organizational justice and job satisfaction among teachers in Bandar Abbas middle school. Procedia-Social and Behavioral Sciences, 5, 1986-1990. doi: 10.1016/j.sbspro.2010.07.401

\title{
Dados dos Autores
}

\author{
Ilse Maria Beuren \\ Universidade Federal de Santa Catarina, Centro Socioeconômico, Campus Universitário, Trindade, 88040-970, Florianópolis, \\ SC, Brasil. E-mail: ilse.beuren@gmail.com \\ Luciana Klein \\ Av. Prefeito Lothário Meissner, 632, Campus III, Jardim Botânico, 80210-170, Curitiba, PR, Brasil. E-mail: \\ lucianaklein.ufpr@gmail.com \\ Flávio Luiz Lara \\ Av. Prefeito Lothário Meissner, 632, Campus III, Jardim Botânico, 80210-170, Curitiba, PR, Brasil. E-mail: \\ flaviolara@ifce.edu.br \\ Lauro Brito de Almeida \\ Av. Prefeito Lothário Meissner, 632, Campus III, Jardim Botânico, 80210-170, Curitiba, PR, Brasil. E-mail: \\ laurobrito52@gmail.com
}

